\title{
Use of a primary care and pharmacy-based model for the delivery of injectable opioid agonist treatment for severe opioid use disorder: a case report
}

\author{
Tyler Wilson MD, Rupi Brar MD, Christy Sutherland MD, Seonaid Nolan MD
}

n Cite as: CMAJ 2020 February 3;192:E115-7. doi: 10.1503/cmaj.190732

A

48-year-old man presented to his primary care clinic, requesting treatment for severe opioid use disorder (as defined by the Diagnostic and Statistical Manual of Mental Disorders, 5th Edition). He reported using intravenous (IV) heroin for the past 20 years and was currently injecting one-quarter of a gram daily. His lifetime overdose history was unknown, but the patient reported 9 overdoses in the few months preceding presentation to the clinic. The patient also reported injecting crystal methamphetamine with heroin twice per week for the past 8 years. He had active nicotine use disorder and an alcohol use disorder in sustained remission. The patient's medical history included posttraumatic stress disorder from childhood trauma, and depression. His daily medications included venlafaxine $75 \mathrm{mg}$, mirtazapine $15 \mathrm{mg}$ and prazosin $3 \mathrm{mg}$ (with which he was intermittently compliant). He had no allergies, was homeless and relied on criminal activities to support his addiction. He had used methadone twice (maximum daily dose of $110 \mathrm{mg}$, maximum duration 6 years) as well as buprenorphine-naloxone (maximum daily dose of $16 \mathrm{mg}, 3$ months' duration) and slowrelease oral morphine (maximum daily dose of $140 \mathrm{mg}$, 2 months' duration). The patient had also previously been offered counselling but did not engage with this.

On physical examination, the patient was seated comfortably in the outpatient clinic and was alert, with no overt signs of intoxication or withdrawal. Investigations showed a normal complete blood count and renal and liver profile. A urine drug test completed 6 weeks previously was positive for fentanyl, opiates and amphetamines.

Given the patient's previous unsuccessful attempts with oral pharmacotherapy and high overdose risk, injectable opioid agonist therapy with hydromorphone was started. The patient's primary care physician (who has experience in addiction medicine) prescribed induction, which was completed in clinic (under nursing supervision), with subsequent transition to a community pharmacy for ongoing witnessed administration. For details of the induction protocol, see Box 1. After day 4, the patient's dose was titrated by $10 \mathrm{mg}$ IV daily to control cravings and withdrawal.

\section{KEY POINTS}

- Injectable opioid agonist therapy can be an effective treatment option for individuals with severe opioid use disorder.

- Induction of injectable opioid agonist therapy was feasibly integrated into a primary care clinic setting.

- Dose administration and monitoring of injectable opioid agonist therapy was safely transitioned to a designated community pharmacy upon dose stabilization.

- Deintensification of therapy can be considered after stabilization with injectable opioid agonist therapy.

Subsequently, he was transitioned to injectable opioid agonist therapy at a designated community pharmacy, where a pharmacist completed a pre- and post-dose assessment, and witnessed administration. However, the patient continued to engage with his primary care clinic for regular assessments, education on harm reduction and ongoing primary care needs. Stabilization occurred about 1 month after induction on hydromorphone $210 \mathrm{mg}$ IV twice daily and $300 \mathrm{mg}$ slow-release oral morphine in the evening. After stabilization, the patient reported that he had no illicit opioid use. Urine drug tests, although consistently positive for crystal methamphetamine, were negative for fentanyl (and all other substances) 2 weeks after induction of injectable opioid agonist therapy, and for 6 months thereafter. The patient also adhered to his antidepressant medications, reported improved mental health, ceased survival crime, transitioned into stable housing and started working in a low-barrier employment program.

Three months after induction, the patient decided (without medical support) to discontinue his second dose of IV hydromorphone so as to facilitate employment. He was prescribed $210 \mathrm{mg}$ IV hydromorphone in the morning and $300 \mathrm{mg}$ of slow-release oral morphine before bedtime for a period of 18 months. After this, the patient chose to transition to buprenorphine-naloxone to accommodate a planned vacation. Upon his return, the patient was eager to restart on IV hydromorphone but relapsed to frequent fentanyl use. He is currently being restarted on injectable opioid agonist therapy. 
Box 1: Induction and stabilization injectable opioid agonist protocol

\begin{tabular}{|c|c|c|c|c|c|c|}
\hline & Day 1 & Day 2 & Day 3 & Day 4 & Day 5 & $\begin{array}{c}\text { Day } 39 \\
\text { (max dose) }\end{array}$ \\
\hline Site of administration & Clinic & Clinic & Clinic & Pharmacy & Pharmacy & Pharmacy \\
\hline $\begin{array}{l}\text { Hydromorphone } \\
\text { morning administration } \\
\text { IM or IV }^{*}\end{array}$ & $\begin{array}{l}15 \mathrm{mg}+30 \mathrm{mg} \text { if } \\
\text { initial dose tolerated }\end{array}$ & $\begin{array}{l}50 \mathrm{mg}+30 \mathrm{mg} \text { if } \\
\text { initial dose tolerated }\end{array}$ & $\begin{array}{c}100 \mathrm{mg} \text { as single } \\
\text { dose }\end{array}$ & $\begin{array}{c}110 \mathrm{mg} \text { as single } \\
\text { dose }\end{array}$ & $\begin{array}{c}120 \mathrm{mg} \text { as single } \\
\text { dose }\end{array}$ & $\begin{array}{l}210 \mathrm{mg} \text { as } \\
\text { single dose }\end{array}$ \\
\hline $\begin{array}{l}\text { Hydromorphone } \\
\text { evening administration } \\
\text { IM or IV }^{\star}\end{array}$ & $\begin{array}{l}45 \mathrm{mg}+30 \mathrm{mg} \text { if } \\
\text { initial dose tolerated }\end{array}$ & $\begin{array}{l}80 \mathrm{mg}+20 \mathrm{mg} \text { if } \\
\text { initial dose tolerated }\end{array}$ & $\begin{array}{c}100 \mathrm{mg} \text { as single } \\
\text { dose }\end{array}$ & $\begin{array}{c}110 \mathrm{mg} \text { as single } \\
\text { dose }\end{array}$ & $\begin{array}{c}120 \mathrm{mg} \text { as single } \\
\text { dose }\end{array}$ & $\begin{array}{l}210 \mathrm{mg} \text { as } \\
\text { single dose }\end{array}$ \\
\hline $\begin{array}{l}\text { Slow-release oral } \\
\text { morphine evening dose } \\
\text { (to prevent withdrawal } \\
\text { overnight) }\end{array}$ & $140 \mathrm{mg}$ & $140 \mathrm{mg}$ & $180 \mathrm{mg}$ & $180 \mathrm{mg}$ & $180 \mathrm{mg}$ & $\begin{array}{l}300 \mathrm{mg} \text { as } \\
\text { single dose }\end{array}$ \\
\hline
\end{tabular}

Note: IM = intramuscular, IV = intravenous.

*The patient is observed for 30 minutes between dose injections (when 2 doses are given) and for 15 minutes after the last injection to ensure tolerability. Intravenous doses were self-injected.

\section{Discussion}

Opioid use disorder is an increasingly prevalent and costly disease in Canada, accounting for a high degree of morbidity, mortality and health care utilization. ${ }^{1}$ Between 2016 and 2018, more than 11500 Canadians died from an opioid-related cause. ${ }^{1}$ Furthermore, 4460 opioid-related deaths occurred in Canada in 2018 alone. This represents a $47 \%$ increase compared with $2016 .{ }^{1}$ Of these deaths, 73\% involved fentanyl (or its analogs) in 2018 (compared with 54\% in 2016). ${ }^{1}$ Other than death, patients with opioid use disorder also experience extensive harms, including violence; nonfatal overdose; and the acquisition of viral infections (e.g., hepatitis C, HIV), skin and soft tissue infections, and infective endocarditis., ${ }^{2,3}$

Accordingly, the burden associated with opioid use disorder has a substantial impact on Canada's health care system, with an estimated 4699 hospital admissions occurring nationally as a result of opioid poisoning in 2014 (a 30\% increase compared with 2007). ${ }^{4}$ Opioid-related presentations to the emergency department are also on the rise. In Alberta and Ontario, respectively, about 2377 and 1143 presentations to the emergency department were for opioid-related causes in $2014 .{ }^{4}$ In Alberta, this reflects a $53 \%$ increase compared with $2010 .{ }^{4}$

There is an urgent need to improve access to evidence-based addiction treatment for opioid use disorder. ${ }^{2,3,5}$ Canada recently adopted national guidelines for the treatment of the disorder. ${ }^{2}$ Included in these guidelines is the need to improve access to alternative treatment options (including both slow-release oral morphine and injectable opioid agonist therapy) for individuals who are unsuccessful on first-line therapies. ${ }^{2}$ Furthermore, the guidelines urge health care providers to match treatment intensity to disease severity (similar to the treatment of all other chronic, relapsing conditions). Although oral opioid agonist therapy (e.g., buprenorphine-naloxone or methadone) can be effective treatments for many individuals with opioid use disorder, retention remains persistently low, with British Columbia data showing that only $32 \%$ of patients were retained on methadone at 1 year. ${ }^{6}$
The use of injectable opioid agonist therapy for individuals with severe opioid use disorder who have been unsuccessful on previous oral opioid agonist therapy is evidence based, with 1 Canadian randomized clinical trial showing improved treatment outcomes among individuals who received injectable diacetylmorphine (heroin) compared with oral methadone. ${ }^{7}$ These outcomes included significantly higher treatment retention at 1 year follow-up ( $88 \%$ v. $54 \%$ respectively, $p<0.001$ ), as well as a reduction in use of illicit drugs and in other illegal activity. ${ }^{7}$ These findings are supported by a Cochrane review of 8 randomized controlled trials of injectable diacetylmorphine therapy, which showed an increase in addiction treatment retention when compared with oral methadone, as well as less criminal activity and a reduction in incarceration and the use of illicit substances. ${ }^{8}$ Subsequent to this, a Canadian study showed injectable hydromorphone to be noninferior to injectable diacetylmorphine for the treatment of individuals with severe opioid use disorder. ${ }^{9}$ Given these results, injectable hydromorphone therapy is increasingly being prescribed as a treatment option to patients with severe opioid use disorder across Canada.

At the same time, consideration is being given to the appropriate model of care to support this treatment option. Clinics specializing in injectable opioid agonist therapy (such as Crosstown Clinic, located in Vancouver, BC) have successfully reduced illicit opioid use and improved engagement in addiction treatment, ${ }^{7,9}$ but limitations prevent their replication and expansion, both provincially and nationally. More specifically, a clinic specializing in injectable opioid agonist therapy requires a large amount of infrastructure, including physical space and staff for the facility. Not surprisingly, this is associated with a costly price tag. Embedding administration of injectable opioid agonist therapy within primary care clinics and designated pharmacies negates the need for new infrastructure and can be operationalized at relatively low cost, relying instead on already established structures and processes. Such a set-up may be particularly attractive in rural settings, where there may be fewer resources and patients are 
dispersed over a wide geographic area. Furthermore, integrating injectable opioid agonist therapy within a primary care setting also allows for an individual prescriber (or team of health care providers) to address outstanding primary care needs (beyond an individual's addiction treatment needs) in parallel, and provides much-needed continuity of care for patients as they transition between different opioid agonist therapy options (e.g., injectable and oral) over time..$^{10}$ It is important to note that, realistically, not all primary care clinics or community pharmacies are positioned to have staff adequately trained to provide injectable opioid agonist therapy, but the existence of a collection of community clinics and pharmacies with this skill set would allow for the referral of appropriate patients within a community setting to receive injectable opioid agonist therapy. Specific details regarding the different models of administration of injectable opioid agonist therapy and required training can be found in the $\mathrm{BC}$ Centre on Substance Use's guidance document on injectable opioid agonist therapy. ${ }^{10}$

In this case, administration of injectable opioid agonist therapy resulted in a number of positive short-term outcomes for a patient with severe opioid use disorder, including cessation of illicit opioid use, a reduction in criminal activity, transition into stable housing and employment. Accordingly, adoption of a primary care and pharmacy-based model for medication administration and monitoring may be a feasible way to expand injectable opioid agonist therapy as a treatment option for individuals with severe opioid use disorder. Further research, including randomized controlled trials, to rigorously evaluate the efficacy of a a primary care and pharmacy-based model of administration of injectable opioid agonist therapy (and to provide definitive recommendations regarding its use for treating opioid use disorder) should be implemented in a timely manner to help address Canada's opioid epidemic.

\section{References}

1. Special Advisory Committee on the Epidemic of Opioid Overdoses. National report: apparent opioid-related deaths in Canada. Ottawa: Public Health Agency of Canada; 2019.

2. Bruneau J, Ahamad K, Goyer MÈ, et al. Management of opioid use disorders: a national clinical practice guideline. CMAJ 2018;190:E247-57.

3. A Guideline for the clinical management of opioid use disorder. Vancouver: British Columbia Centre on Substance Use: 2017. Available: www.bccsu.ca/care -guidance-publications/ (accessed 2020 Jan. 8).

4. Hospitalizations and emergency department visits due to opioid poisoning in Canada. Ottawa: Canadian Institute for Health Information and Canadian Centre on Substance Abuse; 2016.

5. Goel N, Adams D, Ali F, et al. National consultation on the Section 56 exemption requirement for methadone prescribing. Ottawa: Health Canada; 2018.

6. BC opioid substitution treatment system: performance measures 2014/20152015/2016. Victoria (BC): Office of the Provincial Health Officer; 2017.

7. Oviedo-Joekes E, Brissette S, Marsh DC, et al. Diacetylmorphine versus methadone for the treatment of opioid addiction. N Engl J Med 2009;361:777-86.

8. Ferri M, Davoli M, Perucci CA. Heroin maintenance for chronic heroin-dependent individuals. Cochrane Database Syst Rev 2011;(12):CD003410.

9. Oviedo-Joekes E, Guh D, Brissette S, et al. Hydromorphone compared with diacetylmorphine for long-term opioid dependence: a randomized clinical trial. JAMA Psychiatry 2016;73:447-55.

10. Guidance for injectable opioid agonist treatment for opioid use disorder. Vancouver (BC): British Columbia Centre on Substance Use; 2017. Available: www. bccsu.ca/care-guidance-publications/ (accessed 2020 Jan. 8).
Competing interests: Christy Sutherland is the medical director of PHS Community Services Society, a nonprofit that provides housing and harm reduction services. Dr. Sutherland created and implemented the injectable opioid agonist therapy program for PHS Community Services Society. Seonaid Nolan is supported by a Michael Smith Foundation for Health Research Award and the University of British Columbia's Steven Diamond Professorship in Addiction Care Innovation. No other competing interests were declared.

This article has been peer reviewed.

The authors have obtained patient consent.
Affiliations: British Columbia Centre on Substance Use (Wilson, Brar, Sutherland, Nolan); Department of Medicine (Brar, Nolan, Sutherland), University of British Columbia, St. Paul's Hospital, Vancouver, BC

Contributors: All of the authors contributed to the conception and design of the work, drafted the manuscript, revised it critically for important intellectual content, gave final approval of the version to be published and agreed to be accountable for all aspects of the work.

Correspondence to: Seonaid Nolan, Seonaid.nolan@bccsu.ubc.ca 